\title{
Analysis of circulating tumour cell and the epithelial mesenchymal transition (EMT) status during eribulin-based treatment in 22 patients with metastatic breast cancer: a pilot study
}

Yoshiya Horimoto ${ }^{1,2^{*} \mathbb{D}}$, Emi Tokuda ${ }^{1}$, Fumi Murakami ${ }^{1}$, Toshitaka Uomori ${ }^{1}$, Takanori Himuro ${ }^{1}$, Katsuya Nakai ${ }^{1}$, Gotaro Orihata' ${ }^{1}$ Kotaro lijima', Shinsaku Togo ${ }^{3}$, Hideo Shimizu' ${ }^{1}$ and Mitsue Saito'

\begin{abstract}
Background: Liquid biopsy approaches, such as measuring circulating tumour cells (CTCs), have recently been introduced in several clinical studies. However, the development of CTCs as a predictive marker for treatment effects on breast cancer remains an enormous task. We investigated CTCS, including epithelial mesenchymal transition (EMT) status, from metastatic breast cancer patients who had received eribulin-based treatment, which reportedly suppresses EMT as a means of tumour suppression. Our aim was to test the possibility of this method serving as a tool predicting eribulin efficacy.

Methods: Twenty-two patients were enrolled and peripheral blood samples were collected before eribulin treatment. CTCs were then examined using a Microfluidic Chip device. CTCs positive for vimentin and pan-cytokeratin were defined as mesenchymal and epithelial CTCs, respectively. Progression-free survival (PFS) and clinical response were assessable in 20 and 18 patients, respectively, in relation to the number of CTCs.

Results: Numbers of total CTCs were significantly increased in patients with progressive disease during treatment $(p=0.006)$. Median PFS was 14.6 weeks and patients with more total and mesenchymal CTCs at baseline had significantly shorter PFS ( $p=0.0013$ and 0.013 , respectively). Multivariate logistic regression analysis revealed small number of total baseline CTCs and long disease-free survival to be related to long PFS ( $p=0.0004$ and 0.020 , respectively).

Conclusions: Our data suggest that determining both mesenchymal and epithelial CTCs at baseline might be a good tool for predicting eribulin responsiveness. Evaluation of mesenchymal CTC can be considered as a parameter in larger studies, while most clinical trials are currently employing only the detection of the epithelial cellular adhesion molecule (EpCAM).
\end{abstract}

Keywords: Breast cancer, Circulating tumour cell, Liquid biopsy, Eribulin, Epithelial mesenchymal transition

\footnotetext{
*Correspondence: yoshiyahorimoto@hotmail.com

${ }^{1}$ Department of Breast Oncology, Juntendo University School

of Medicine, 2-1-1 Hongo, Bunkyo-ku, Tokyo 113-0033, Japan

Full list of author information is available at the end of the article
} 


\section{Background}

\section{CTC analysis in breast cancer research}

Liquid biopsy approaches, such as measuring circulating tumour cells (CTCs) and DNA, have recently been introduced in clinical studies on a broad range of cancer types. Several studies have shown high numbers of CTCs to correlate with poorer outcomes in patients with both early and metastatic breast cancer (MBC), such that CTCs are now a relatively well established prognostic marker [1-3]. Moreover, two studies revealed some patients to have HER2 positive CTCs, despite their primary tumours being HER2-negative $[4,5]$ and such information might aid in making treatment decisions. However, there are still many problems, including standardisation of analysis since laboratories employ different techniques, before CTC analysis can be introduced into routine clinical practice. Also, evidence supporting CTCs as a marker predicting treatment effects in breast cancer remains insufficient $[2,4,6]$.

\section{Improvement of technology in CTC analysis}

The CellSearch ${ }^{\circledR}$ System has become the most widely used technique for CTC analysis in both pre-clinical and clinical studies [7]. This system captures CTCs using antibodies against epithelial cellular adhesion molecule (EpCAM) and several epithelial cell surface markers, including CK8, 18 and 19. EpCAM is a conventional marker well-known to be expressed in epithelial-origin cancer cells. However, a growing body of evidence has revealed that EpCAM is not expressed in all CTCs [8-10]. A subpopulation of CTCs with decreased levels of epithelial markers might escape from EpCAM-based detection [8] and epithelial-to-mesenchymal transition (EMT) and could thereby be a major reason for this phenomenon [6, 8, 11-15].

To overcome this defect in EpCAM-based detection, technologies for CTC analysis have advanced and the EMT status of CTCs can now also be assessed [14, 16, 17]. For instance, changes in EMT status during treatments of individual patients with MBC were reported [6]. Several studies have shown improved CTC detection by employing EMT markers in addition to epithelial markers $[18,19]$.

\section{Eribulin treatment in $\mathrm{MBC}$ patients}

$\mathrm{MBC}$ is essentially incurable although many treatment options including endocrine agents and chemotherapy are available. Individual MBCs respond differently even to the same treatments. Therefore, we often encounter difficulties in choosing the most appropriate treatments with optimal timing for individual patients. Thus, predictive markers for therapeutic efficacy are eagerly awaited.
Eribulin is a microtubule-depolymerising agent and was introduced as an option for MBC patients, following confirmation of clinically meaningful improvement in overall survival [20]. Eribulin was also recently approved by the Food and Drug Administration of the United States as a liposarcoma treatment. Interestingly, the suppression of EMT might reportedly be the mechanism by which eribulin regulates breast cancer progression both in vitro and in vivo $[21,22]$.

We investigated CTCs in MBC patients who had received eribulin, employing an established system that can evaluate EMT status. The current study aimed to test the possibility of this method serving as a tool for predicting eribulin efficacy. We are also interested in whether the EMT status of CTCs is suppressed in response to eribulin treatment.

\section{Methods}

\section{Patients and treatments}

Twenty-two patients with metastatic/stage IV breast cancer, who had started eribulin-based treatments at our department during the January through December 2017 period were enrolled. Seventeen patients developed metastatic disease after undergoing curative surgery for primary breast cancer, while 5 had Stage IV disease. Clinicopathological features of the 22 patients are shown in Table 1. Mean patient age at the time of starting eribulin was 57 (range 38-81) years. The intrinsic subtype rates of the primary tumours were: luminal-HER2(-) $64 \%$, luminal-HER2 $(+)$ 9\%, HER2 type 5\%, and triple negative (TN) 23\%. Median disease-free-survival after curative surgery was 78 months (range 12-125). Metastatic sites were bone (59\%), liver (36\%), the lungs (32\%), pleura (9\%), brain (9\%) and others (9\%). Eribulin was administrated as the first, second, third/more line of chemotherapy for metastatic disease in 36\%, 55\% and 9\% of patients, respectively.

Eribulin mesylate was administered at the standard dose of $1.4 \mathrm{mg} / \mathrm{m}^{2}$ on day 1 and day 8 . During treatment, drug doses were gradually reduced as needed, such as in cases with neutropenia. Anti-HER2 drugs, such as Trastuzumab and Pertuzumab, were simultaneously administered according to the HER2 status of the patient's tumour.

Peripheral blood $(10 \mathrm{ml})$ samples were collected before eribulin treatment. The samples were sent to the Nihon Gene Research Laboratories (Japan) within $24 \mathrm{~h}$ for analysis of CTCs. CTCs were re-examined in some patients when treatment effects were evaluated (details presented in the CTC analysis section). This study was carried out with approval from the ethics committee of Juntendo University Hospital (no: 16-139) and all samples were 
Table 1 Clinicopathological features of 22 patients

\begin{tabular}{|c|c|}
\hline$n$ & 22 \\
\hline Median age (range) & $57(38-81)$ \\
\hline \multicolumn{2}{|c|}{ Characteristics of primary tumour } \\
\hline \multicolumn{2}{|l|}{ Histology } \\
\hline IDC (NST) & $18(82 \%)$ \\
\hline Special type & $4(18 \%)$ \\
\hline \multicolumn{2}{|l|}{ Grade } \\
\hline High & $5(23 \%)$ \\
\hline Low-intermediate & $13(59 \%)$ \\
\hline Unknown & $4(18 \%)$ \\
\hline \multicolumn{2}{|l|}{ Intrinsic subtype ${ }^{\mathrm{b}}$} \\
\hline Luminal-HER2(-) & $13(59 \%)$ \\
\hline Luminal-HER2(+) & $3(14 \%)$ \\
\hline HER2 & $1(5 \%)$ \\
\hline Triple negative & $5(23 \%)$ \\
\hline DFS (months) ${ }^{c}$ (range) & $78(12-125)$ \\
\hline \multicolumn{2}{|l|}{ Metastatic site } \\
\hline Bone & $13(59 \%)$ \\
\hline Liver & $8(36 \%)$ \\
\hline Lungs & $7(32 \%)$ \\
\hline Pleura & $2(9 \%)$ \\
\hline Brain & $2(9 \%)$ \\
\hline Others & $2(9 \%)$ \\
\hline \multicolumn{2}{|c|}{ Number of previous chemotherapies for metastatic disease } \\
\hline 0 & $8(36 \%)$ \\
\hline $1-2$ & $12(55 \%)$ \\
\hline $3-$ & $2(9 \%)$ \\
\hline
\end{tabular}

DFS disease-free survival, IDC invasive ductal carcinoma, NST non-special type

a At the time of starting eribulin

${ }^{b}$ HER2 overexpression was defined as IHC (3+) or FISH (+)

c For 17 patients who underwent curable surgery for the primary tumour

collected after obtaining written informed consent from the patients.

\section{Evaluations of clinical response and progression free survival (PFS)}

Clinical responses were evaluated with radiographic images based on the RECIST (version 1.1) guideline [23], which defines complete response (CR), partial response $(\mathrm{PR})$, stable disease (SD), and progressive disease (PD). Analysis criteria are listed in Fig. 1. Following the baseline CTC assessment, 2 patients were withdrawn and did not undergo evaluation of treatment effects: 1 patient developed metastasis involving the central nerve system just 1 week after the first eribulin administration and the other patient suffered continuous bone marrow suppression. Evaluations were basically conducted after three treatment courses but the timing differed among cases, since it was determined based on individual disease states. One patient had only non-measurable metastatic disease, i.e. pleural effusion. Another patient had only bone metastases appearing as sclerotic changes on computed tomography scans. Thus, we excluded these 2 patients from the evaluation of clinical response but they were included in the population for PFS analysis.

\section{CTC analysis}

CTCs were examined using a Microfluidic Chip device at Nihon Gene Research Laboratories (Japan). This commercially available system captures and isolates rare CTCs from blood samples with 56,320 wells, based on their size and deformability differences from blood cells [16]. Thereby, this system provides very high efficiency. Fluorescence images of cells, including EMT markers, can be obtained using an automated staining and scanning system.

CTCs positive for vimentin and pan-cytokeratin were defined as mesenchymal (mCTCs) and epithelial (eCTCs), respectively. Representative $\mathrm{mCTC}$ and eCTC cases in our cohort are shown in Fig. 2. Case 1 is positive only for Vimentin and was thus judged as having mCTC. Case 2 is positive for cytokeratin and was thus defined as having eCTC. CTCs positive for both cytokeratin and vimentin, observed in a few cases, were defined as mCTCs in the current study, as indicated in Fig. 2. Patients, who had at least four CTCs per $4 \mathrm{ml}$ in total or any $\mathrm{mCTCs}$ at the beginning of the treatment, underwent the second CTC test when treatment effects were evaluated.

\section{Statistical analysis}

Statistical analyses were performed using JMP 11.2.1 statistical software (SAS Institute Inc., Cary, NC, USA). Associations between clinicopathological parameters and patient outcomes were evaluated using Fisher's exact test. For comparisons of the median values of changes in the numbers of CTCs during treatments, examinations of paired data were carried out employing the Mann-Whitney U test. Kaplan-Meier curves were estimated and the log-rank test was applied for comparisons of the survival distributions of two populations. The logistic regression model was constructed using the stepwise procedure with the minimal Bayesian information criterion in an attempt to identify predictors of long PFS. Among factors selected by univariate analysis, DFS and total CTCs were selected as full-model variables for the present analysis. A $p<0.05$ was considered to indicate a statistically significant difference.

\section{Results}

mCTCs were frequently observed in patients with TN MBC CTCs were detected in 21 of 22 patients. The median number of total CTCs was 3.0 per $4.0 \mathrm{ml}$. When the 


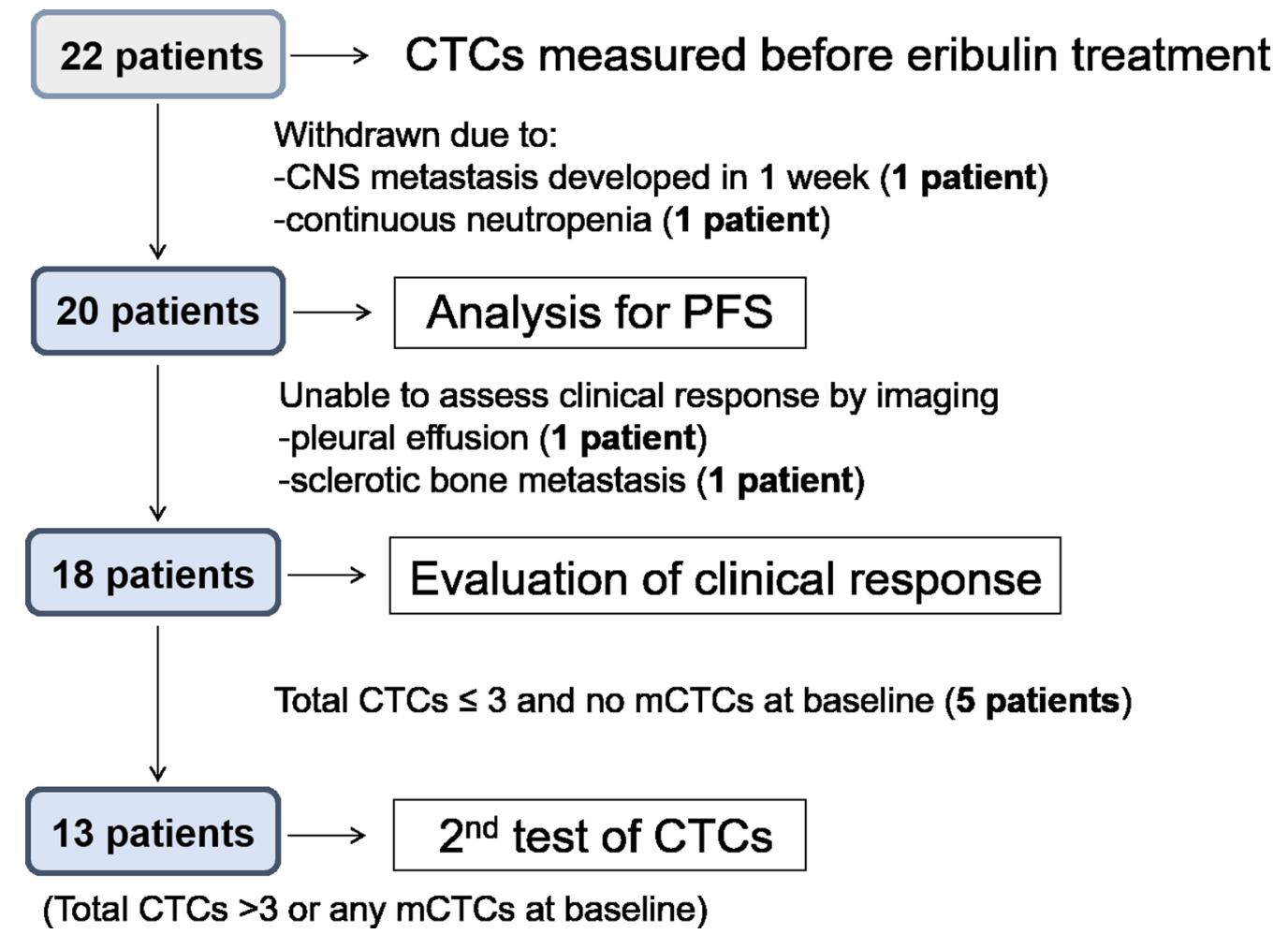

Fig. 1 Flow chart of analysis criteria. PFS was analysed in 20 patients, after 2 had been withdrawn, due to rapid development of CNS metastasis and continuous neutropenia. Of these 20 patients, we were able to evaluate clinical responses based on imaging in 18. Thirteen patients, with at least 4 total CTCs or any mCTCs at baseline, underwent a second CTC test when treatment effects were assessed

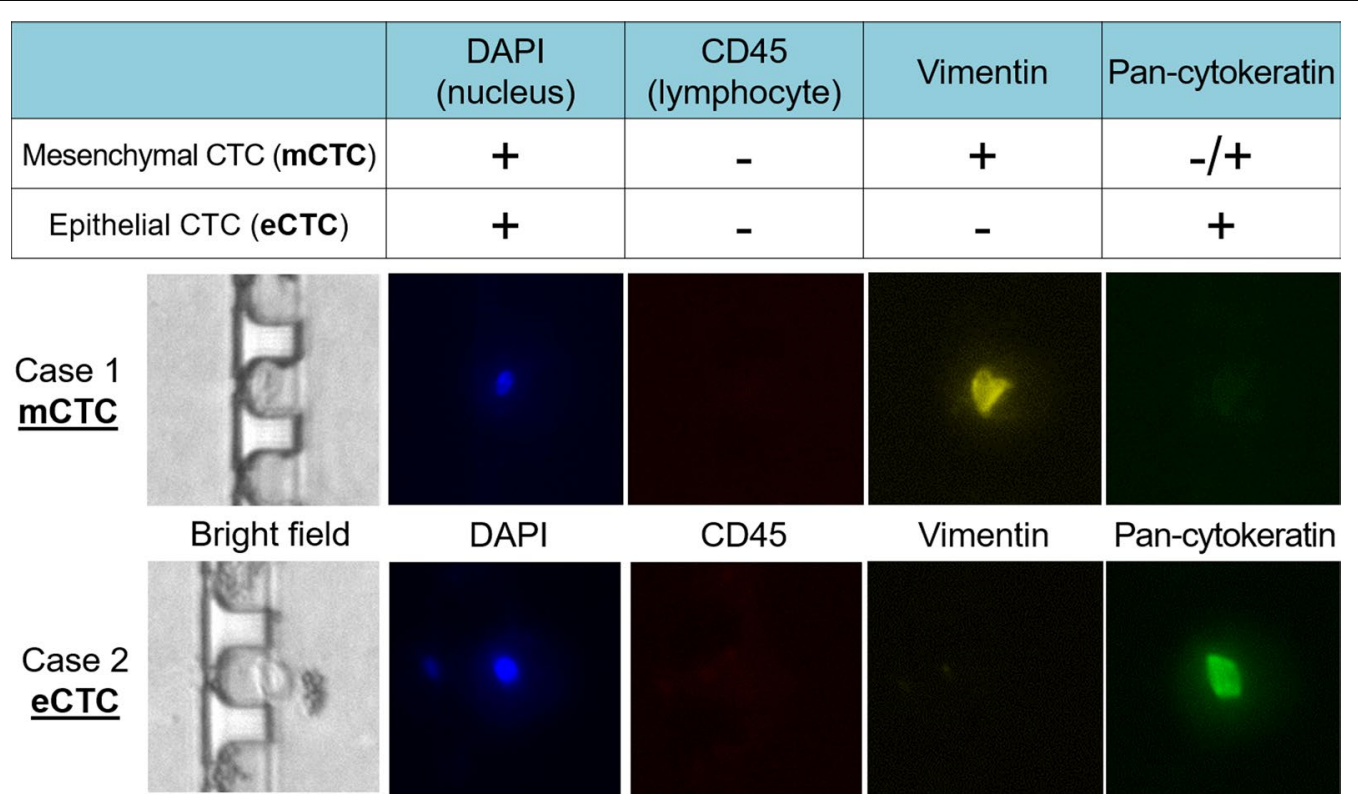

Fig. 2 CTC detection and mCTC and eCTC definitions. CTCs captured and isolated from blood samples were stained and scanned automatically. Representative fluorescence images of mCTC-predominant and eCTC-predominant cases in our cohort are shown 
results were analysed according to intrinsic subtype, there were no differences in total number of CTCs (Additional file 1: Table S1). However, mCTCs were more commonly observed in TN than in other cancer cases and the $\mathrm{mCTC}$ rate was significantly higher in $\mathrm{TN}$ cases than in those with luminal HER2-negative MBC $(\mathrm{p}<0.05)$.

\section{PD group tended to have more total and mCTCs}

Treatment responses in 18 patients were analysed. Rates of PR, SD and PD were 17\% (3 cases), 22\% (4) and 61\% (11), respectively, while there were no CR cases. Clinical benefits, i.e. PR and SD lasting longer than 3 months, were obtained in 7 patients (39\%). When the patients were categorised into two groups, $\mathrm{PR} / \mathrm{SD}$ and $\mathrm{PD}$, total and $\mathrm{mCTC}$ were higher in the PD group (mean 2.3 vs 6.0 and 1.0 vs 3.5 , respectively), although the differences were not statistically significant (Fig. 3a).

\section{Total CTCs increased during treatments in the PD group} Changes in CTCs during treatments were evaluated in 13 patients who underwent the second CTC test. As shown in Fig. 3b, the total CTC number was significantly increased in the PD group $(\mathrm{p}=0.0060)$, with $\mathrm{mCTC}$ showing a similar tendency $(\mathrm{p}=0.073)$. However, in the $\mathrm{PR} / \mathrm{SD}$ cases, no trends were observed across all CTC types.

Furthermore, we investigated changes in the proportions of mCTC and eCTC during treatments. The results, according to clinical response, are shown in Fig. 3c. No significant trend was observed in the mCTC rate, regardless of the change in the pattern of total CTCs. Interestingly, when we focus on patients whose CTCs at baseline were all mCTC (3 patients each in the $\mathrm{PR} / \mathrm{SD}$ and $\mathrm{PD}$ groups), all 3 in the $\mathrm{PR} / \mathrm{SD}$ group showed a decrease in the $\mathrm{MCTC}$ rate, while none of the 3 in the PD group showed a decrease.

\section{Patients with more total CTCs and mCTCs had shorter PFS} PFS was assessed in 20 patients. Median PFS was 14.6 weeks and 3 patients are still receiving treatments. Kaplan-Meier curves for PFS according to CTC types are presented in Fig. 4. Patients with more total CTCs had significantly shorter DFS than those with fewer CTCs $(p=0.0013)$. The same trend was observed for mCTCs with a statistically significant difference $(p=0.013)$. On the other hand, there were no differences according to numbers of eCTCs. When the differences between the total and MCTC groups were compared, the gap was much wider for total CTC than for mCTCs.

\section{Small number of total CTCs and long DFS predict long PFS}

Finally, we sought factors possibly predicting long PFS (6 months or longer). Fisher's exact test revealed that total CTCs (less than 3), mCTCs (less than 2) and DFS (60 months or more) correlated with long PFS, as well as other clinicopathological factors such as age, tumour grade, ER and HER2 status, metastatic site (visceral vs non-visceral), and previous chemotherapy (Additional file 2: Table S2). We then employed a multivariate logistic regression model for total CTCs and DFS, selected by the stepwise procedure. Both total CTCs and DFS were found to be significantly and independently related to long PFS ( $\mathrm{p}=0.0004$ and 0.020 , respectively) (Table 2).

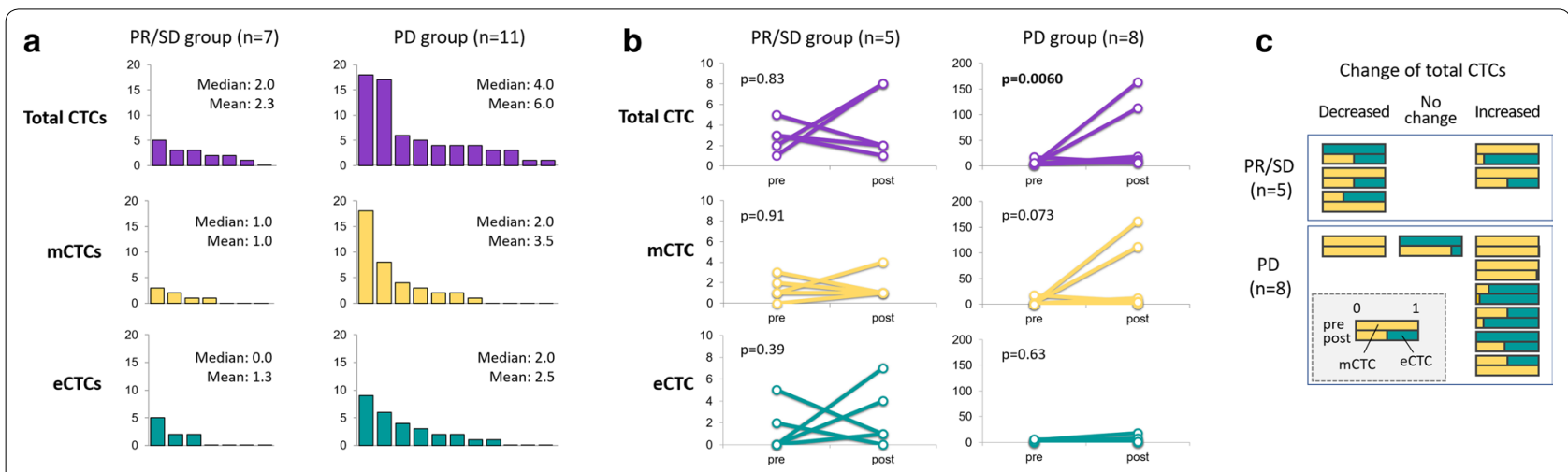

Fig. 3 Baseline CTCs and changes in CTCs during treatments according to treatment effects. a Bar charts show details of baseline CTCs according to treatment responses in 18 patients. Total CTCs, MCTCS and ECTCS are indicated in purple, yellow and green, respectively. $\mathbf{b}$ Line graphs indicate change in CTCs during eribulin administration according to treatment responses in 15 patients, all of whom received a second CTC test. c Proportions of mCTC and eCTC in individual patients during eribulin administration, according to changes in total CTCs, are shown. As indicated in the grey square, the upper bar shows baseline status, the lower bar that during treatment. Yellow and green bars represent the rates of mCTC and eCTC, respectively 

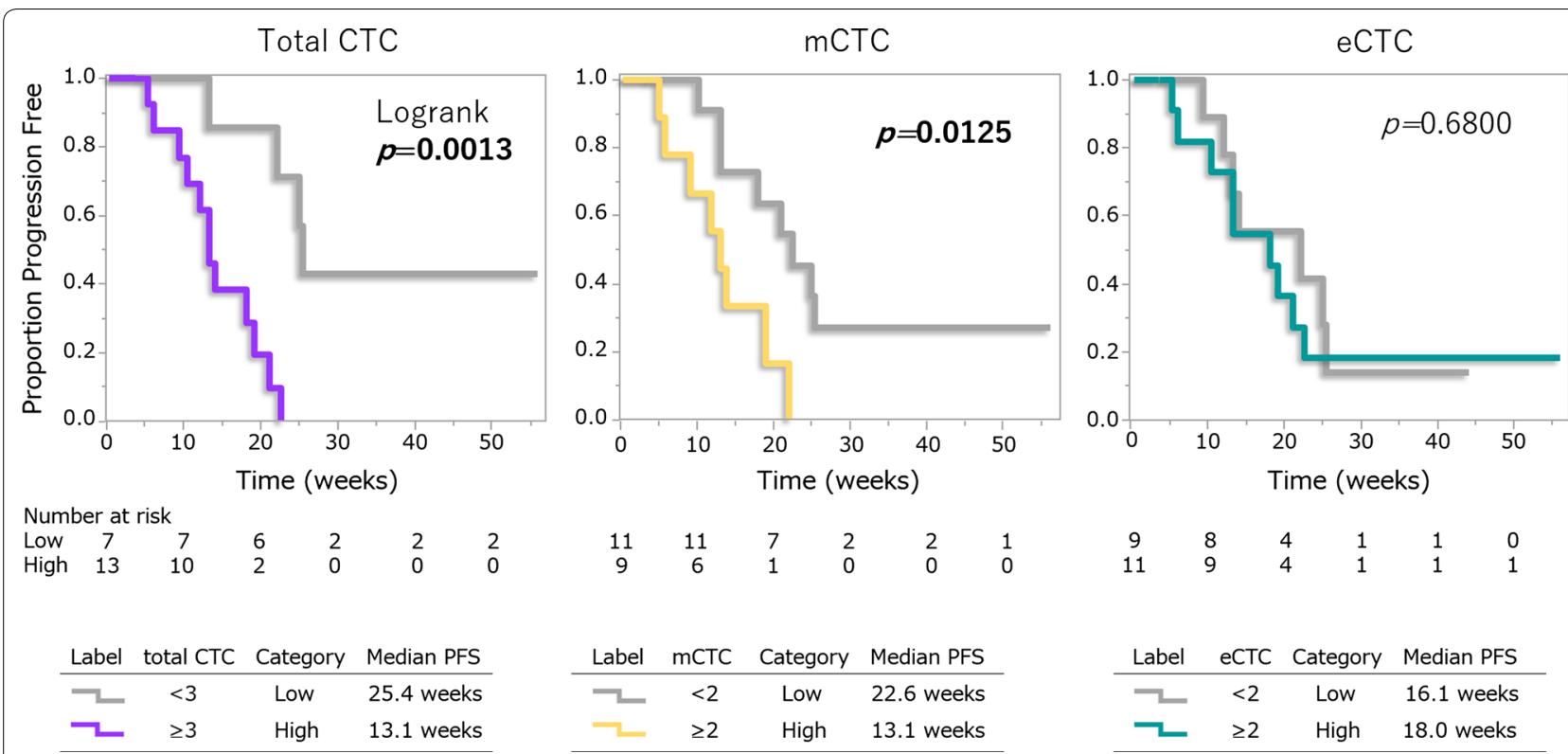

Fig. 4 Kaplan-Meier curves of PFS according to CTC types. Kaplan-Meier curves for PFS in 20 patients according to each CTC type are shown. The log-rank test was applied for comparisons of the survival distributions of the two groups

Table 2 Logistic model for predicting long PFS

\begin{tabular}{lll}
\hline Variables & OR (Cl) & p-value \\
\hline Total CTCs $(<3$ vs 3 or more) & $6.67 \mathrm{E}+14(17.4-\infty)$ & 0.0004 \\
DFS $(60$ months or more vs $<60)$ & $2.32 \mathrm{E}+14(2.78-\infty)$ & 0.020 \\
\hline
\end{tabular}

Italic values indicate significance of $\mathrm{p}$ value $(p<0.05)$

$O R$ odds ratio, $C l$ confidence interval

\section{Discussion}

In the current study, we found that total CTCs, comprised of mCTCs and eCTCs, might predict eribulin efficacy. To our knowledge, the current study is the first to examine the possibility of baseline CTCs serving as a predictive marker specific for eribulin-based treatment. We also monitored changes in the number and EMT status of CTCs during eribulin administration.

Changes in CTC numbers have previously been demonstrated in several studies focusing on other treatments [6, 24, 25]. Yu et al. monitored $11 \mathrm{MBC}$ patients and total CTCs were decreased in responders to various chemotherapeutic regimens [6]. Likewise, in a randomised phase III trial involving $56 \mathrm{MBC}$ patients, a population who showed early reductions in CTCs (detected with the CellSearch System) during chemotherapy had longer PFS [25]. It is understandable that the number of CTCs might simply reflect the total tumour burden in a MBC patient. Our data showed similar trends during eribulin treatment. An increase in
CTC numbers was observed in the PD group, regardless of CTC types.

According to several reports, one of the mechanisms underlying the anti-tumour effects of eribulin might be suppression of EMT [21, 22]. Changes in E-cadherin subcellular localisation and reversal of EMT were demonstrated in response to eribulin in breast cancer cell lines [21]. Yoshida et al. showed that eribulin reversed the EMT phenotype in an in vivo model, resulting in suppression of tumour metastasis [22]. Thus, we initially expected that mCTCs would be decreased in responders to eribulin. Indeed, in the 6 patients whose CTCs were $100 \% \mathrm{mCTCs}$ at baseline, the $\mathrm{mCTC}$ rate was decreased in the three with PR/SD (Fig. 3c), while the three with PD showed no changes. However, overall, no changes or even trends were observed in either the absolute number or the rate of $\mathrm{mCTCs}$ rising and decreasing in the PR/SD group (Fig. 3b, c). Therefore, based on our data, we could not reach a conclusion regarding this issue.

Whether regulation of EMT in CTCs contributes to patient outcomes is still unknown since the opposite phenomenon, MET (mesenchymal epithelial transition), is considered to occur when CTCs develop homing to a distant organ [26]. Further studies are clearly needed to examine this issue in detail.

Patients with more total CTCs and mCTCs had shorter PFS. Our data correlate with those of previous reports showing elevated baseline CTCs to be a predictive marker for poor outcomes after a variety of chemotherapeutic regimens, although most such large trials 
employed EpCAM-based detection [2, 24, 25, 27]. Our data clearly showed no differences in PFS according to eCTC number (Fig. 4). Whether the observed difference is due to eribulin treatment is as yet unknown.

Meanwhile, patients with more total CTCs and mCTCs had significantly shorter DFS than those with fewer CTCs. When the differences in PFS between the two groups, based on total and mCTCs, were compared, the gap was much wider for total CTCs (Fig. 4). This observation suggests that measuring mCTCs only is not sufficient to predict PFS. Instead, assessing total CTCs, based on mCTC and eCTC, might be the optimal approach.

We believe that assessing mCTCs, in addition to eCTCs, would improve the current EpCAM-based analysis, allowing it to serve as a tool for assessing patient outcome and treatment effects.

Limitations of the current study were lack of a control group and the small number of cases. Thus, as a next step, our approach of assessing mCTCs and eCTCs should be introduced in randomised prospective studies, comparing eribulin with other forms of chemotherapy, with a large number of patients.

\section{Conclusions}

Our data suggest that determinations of both mCTCs and eCTCs at baseline provide a good tool for predicting eribulin responsiveness and our observations also suggest that adding $\mathrm{mCTC}$ evaluation to the treatment selection process for $\mathrm{MBC}$ might merit examination in future studies. In summary, this approach may also be worth incorporating into ongoing large trials mostly employing EpCAM-based methods only.

\section{Additional files}

Additional file 1: Table S1. Number of CTCS according to intrinsic subtype.

Additional file 2: Table S2. Univariate analysis of factors related to long PFS.

\section{Abbreviations}

CTCs: circulating tumour cells; CR: complete response; DFS: disease-free survival; eCTCs: epithelial circulating tumour cells; EMT: epithelial mesenchymal transition; IDC: invasive ductal carcinoma; MBC: metastatic breast cancer; mCTCs: mesenchymal circulating tumour cells; NST: non-special type; PD: progressive disease; PFS: progression-free survival; PR: partial response; SD: stable disease; TN: triple negative.

\section{Authors' contributions}

Conception and design: YH, ET. Development of methodology: YH, ET. Acquisition of data (enrolled and managed patients, etc.): YH, ET, FM, TU, TH, KN, GO, KI. Analysis and interpretation of data (e.g. statistical analysis): YH. Writing, review, and/or revision of the manuscript: YH, ET, FM, TU, TH, KN, GO, KI, ST, HS, MS. Study supervision: ST, MS. All authors read and approved the final manuscript.

\begin{abstract}
Author details
1 Department of Breast Oncology, Juntendo University School of Medicine, 2-1-1 Hongo, Bunkyo-ku, Tokyo 113-0033, Japan. ${ }^{2}$ Department of Pathology and Oncology, Juntendo University School of Medicine, 2-1-1 Hongo, Bunkyo-ku, Tokyo 113-0033, Japan. ${ }^{3}$ Department of Respiratory Medicine, Juntendo University School of Medicine, 2-1-1 Hongo, Bunkyo-ku, Tokyo 113-0033, Japan.
\end{abstract}

\section{Acknowledgements}

We are grateful to all study participants and to the nurses at our outpatient clinic for their efforts. We sincerely appreciate Dr. Bierta Barfod for her help with the language editing. We also thank Dr. Kaoru Mogushi of Diagnostics and Therapeutics of Intractable Diseases at Juntendo University Graduate School of Medicine for his advice on the statistical analysis.

\section{Competing interests}

The authors declare that they have no competing interests.

\section{Availability of data and materials}

Data sharing not applicable to this article as no datasets were analysed during the current study.

\section{Consent for publication}

Not applicable.

\section{Ethics approval and consent to participate}

All procedures performed in studies involving human participants were in accordance with the ethical standards of the ethics committee of Juntendo University Hospital (no: 16-139) and with the 1964 Helsinki declaration and its later amendments or comparable ethical standards. Informed consent was obtained from all individual participants included in the study.

\section{Funding}

This study was funded by a Japan Society for the Promotion of Science Grantin-Aid for Scientific Research (Grant Number JP15K10069).

\section{Publisher's Note}

Springer Nature remains neutral with regard to jurisdictional claims in published maps and institutional affiliations.

Received: 24 July 2018 Accepted: 12 October 2018

Published online: 20 October 2018

\section{References}

1. Bidard F-C, Peeters DJ, Fehm T, Nolé F, Gisbert-Criado R, Mavroudis D, et al. Clinical validity of circulating tumour cells in patients with metastatic breast cancer: a pooled analysis of individual patient data. Lancet Oncol. 2014;15:406-14.

2. Bidard F-C, Michiels S, Riethdorf S, Mueller V, Esserman $L$, Lucci A, et al. Circulating tumor cells in breast cancer patients treated by neoadjuvant chemotherapy: a meta-analysis. J Natl Cancer Instit. 2018;110:560-7.

3. Rossi G, Mu Z, Rademaker AW, Austin LK, Strickland KS, Costa RLB, et al. Cell-free DNA and circulating tumor cells: comprehensive liquid biopsy analysis in advanced breast cancer. Clin Cancer Res. 2018;24:560-8.

4. Ignatiadis $\mathrm{M}$, Rack B, Rothé F, Riethdorf S, Decraene C, Bonnefoi H, et al. Liquid biopsy-based clinical research in early breast cancer: the EORTC 90091-10093 treat CTC trial. Eur J Cancer. 2016;63:97-104.

5. Meng S, Tripathy D, Shete S, Ashfaq R, Haley B, Perkins S, et al. HER-2 gene amplification can be acquired as breast cancer progresses. Proc Natl Acad Sci USA. 2004;101:9393-8.

6. Yu M, Bardia A, Wittner BS, Stott SL, Smas ME, Ting DT, et al. Circulating breast tumor cells exhibit dynamic changes in epithelial and mesenchymal composition. Science (New York, NY). 2013;339:580-4.

7. Allard WJ, Matera J, Miller MC, Repollet M, Connelly MC, Rao C, et al. Tumor cells circulate in the peripheral blood of all major carcinomas but not in healthy subjects or patients with nonmalignant diseases. Clin Cancer Res. 2004;10:6897-904. 
8. Gorges TM, Tinhofer I, Drosch M, Röse L, Zollner TM, Krahn T. Circulating tumour cells escape from EpCAM-based detection due to epithelial-tomesenchymal transition. BMC Cancer. 2012;12:178-278.

9. Mikolajczyk SD, Millar LS, Tsinberg P, Coutts SM, Zomorrodi M, Pham T, et al. Detection of EpCAM-negative and cytokeratin-negative circulating tumor cells in peripheral blood. J Oncol. 2011;2011:252361.

10. Königsberg R, Obermayr E, Bises G, Pfeiler G, Gneist M, Wrba F, et al. Detection of EpCAM positive and negative circulating tumor cells in metastatic breast cancer patients. Acta Oncol. 2011:50:700-10.

11. Bidard F-C, Proudhon C, Pierga J-Y. Circulating tumor cells in breast cancer. Mol Oncol. 2016;10:418-30.

12. Armstrong AJ, Marengo MS, Oltean S, Kemeny G, Bitting RL, Turnbull JD, et al. Circulating tumor cells from patients with advanced prostate and breast cancer display both epithelial and mesenchymal markers. Mol Cancer Res. 2011;9:997-1007.

13. Mego M, Cierna Z, Janega P, Karaba M, Minarik G, Benca J, et al. Relationship between circulating tumor cells and epithelial to mesenchymal transition in early breast cancer. BMC Cancer. 2015;15:533.

14. Polioudaki H, Agelaki S, Chiotaki R, Politaki E, Mavroudis D, Matikas A, et al. Variable expression levels of keratin and vimentin reveal differential EMT status of circulating tumor cells and correlation with clinical characteristics and outcome of patients with metastatic breast cancer. BMC Cancer. 2015;15:399.

15. Hyun K-A, Koo G-B, Han H, Sohn J, Choi W, Kim S-I, et al. Epithelial-to-mesenchymal transition leads to loss of EpCAM and different physical properties in circulating tumor cells from metastatic breast cancer. Oncotarget. 2016:7:24677-87.

16. Gogoi P, Sepehri S, Zhou Y, Gorin MA, Paolillo C, Capoluongo E, et al. Development of an automated and sensitive microfluidic device for capturing and characterizing circulating tumor cells (CTCS) from clinical blood samples. PLoS ONE. 2016:11:e0147400.

17. Satelli A, Brownlee Z, Mitra A, Meng QH, Li S. Circulating tumor cell enumeration with a combination of epithelial cell adhesion moleculeand cell-surface vimentin-based methods for monitoring breast cancer therapeutic response. Clin Chem. 2015;61:259-66.

18. OnstenkW, Kraan J, Mostert B, Timmermans MM, Charehbili A, Smit VT, et al. Improved circulating tumor cell detection by a combined EpCAM and MCAM cell search enrichment approach in patients with breast cancer undergoing neoadjuvant chemotherapy. Mol Cancer Ther. 2015:14:821-7.

19. Mostert B, Kraan J, Sieuwerts AM, van der Spoel P, Bolt-de Vries J, Pragervan der Smissen WJC, et al. CD49f-based selection of circulating tumor cells (CTCS) improves detection across breast cancer subtypes. Cancer Lett. 2012;319:49-55.

20. Cortes J, O'Shaughnessy J, Loesch D, Blum JL, Vahdat LT, Petrakova K, et al. Eribulin monotherapy versus treatment of physician's choice in patients with metastatic breast cancer (EMBRACE): a phase 3 open-label randomised study. The Lancet. 2011;377:914-23.

21. Dybdal-Hargreaves NF, Risinger AL, Mooberry SL. Regulation of E-cadherin localization by microtubule targeting agents: rapid promotion of cortical E-cadherin through p130Cas/Src inhibition by eribulin. Oncotarget. 2018;9:5545-61.

22. Yoshida T, Ozawa Y, Kimura T, Sato Y, Kuznetsov G, Xu S, et al. Eribulin mesilate suppresses experimental metastasis of breast cancer cells by reversing phenotype from epithelial-mesenchymal transition (EMT) to mesenchymal-epithelial transition (MET) states. Br J Cancer. 2014;110:1497-505.

23. Eisenhauer EA, Therasse P, Bogaerts J, Schwartz LH, Sargent D, Ford R, et al. New response evaluation criteria in solid tumours: revised RECIST guideline (version 1.1). Eur J Cancer. 2009:45:228-47.

24. Pierga JY, Hajage D, Bachelot T, Delaloge S, Brain E, Campone M, et al. High independent prognostic and predictive value of circulating tumor cells compared with serum tumor markers in a large prospective trial in first-line chemotherapy for metastatic breast cancer patients. Ann Oncol. 2012;23:618-24.

25. Helissey C, Berger F, Cottu P, Diéras V, Mignot L, Servois V, et al. Circulating tumor cell thresholds and survival scores in advanced metastatic breast cancer: the observational step of the CirCe01 phase III trial. Cancer Lett. 2015:360:213-8.

26. De Craene B, Berx G. Regulatory networks defining EMT during cancer initiation and progression. Nat Rev Cancer. 2013;13:97-110.

27. Cristofanilli M, Budd GT, Ellis MJ, Stopeck A, Matera J, Miller MC, et al. Circulating tumor cells, disease progression, and survival in metastatic breast cancer. New Engl J Med. 2004;351:781-91.
Ready to submit your research? Choose BMC and benefit from:

- fast, convenient online submission

- thorough peer review by experienced researchers in your field

- rapid publication on acceptance

- support for research data, including large and complex data types

- gold Open Access which fosters wider collaboration and increased citations

- maximum visibility for your research: over $100 \mathrm{M}$ website views per year

At $\mathrm{BMC}$, research is always in progress.

Learn more biomedcentral.com/submissions 